\title{
Model-Based Development of the RespiraWorks Ventilator with Modelon Impact
}

\author{
John Batteh ${ }^{1} \quad$ Lixiang Li $^{2} \quad$ Edwin Chiu $^{3} \quad$ Ethan Chaleff $^{4}$ \\ 1,2Modelon Inc., USA, \{john.batteh, lixiang. li \} @modelon.com \\ ${ }^{3,4}$ RespiraWorks, USA, \{edwin, ethan\} @respira.works
}

\begin{abstract}
This paper describes the modeling and simulation of the RespiraWorks ventilator in Modelica with Modelon Impact and the Pneumatics Library. Following a brief overview of the RespiraWorks open-source effort in response to COVID-19, details of the pneumatic modeling effort, including the implementation of new components, are provided in support of the model-based development process. The pneumatics models of several different iterations of the ventilator design are shown. Lastly an overview of the model calibration process is provided, and the model results are compared with experimental data collected from the ventilator prototype.

Keywords: pneumatics, ventilator, COVID-19
\end{abstract}

\section{Introduction}

The COVID-19 global pandemic has resulted in a shortage of critical medical resources. In particular, there has been an acute shortage of ventilators, especially in developing countries. Ventilators are expensive medical devices typically developed over 5-6 years. At the start of the pandemic, three engineers, Ethan Chaleff, Edwin Chiu, and Elizabeth Hillstrom, started an effort to develop a low cost open-source ventilator. Within two weeks, two prototypes had been built in a small Berkeley, CA garage. Within a month, the group incorporated RespiraWorks, a 501(c)(3) non-profit organization. Today RespiraWorks has grown to over 200 volunteers with a range of expertise in 10 countries (RespiraWorks 2021).

From its inception, the RespiraWorks mission is to radically democratize the ventilator. The team set out to create a full-featured ventilator that is affordable and easy to build in countries with developing economies and lowresource communities. With an open-source, IP-free design, organizations can leverage local resources to help their people. The group was motivated to remove money as a barrier for people to obtain life-saving medical equipment and to shift revenue motivation largely to those manufacturing and delivering equipment to those in need.

Many of the ventilator efforts spawned during the pandemic were focused on providing a "bridge" ventilator that provides temporary support until the patient can be placed on a full feature ventilator, reducing but not eliminating reliance on imported ventilators. The RespiraWorks mission is to build a fully-featured, fullycertified medical device capable of advanced respiratory support for patients who may be on a ventilator for days or weeks and whose benefit endures beyond the current crisis. The team is committed to helping manufacturers around the world build the ventilator and has signed a memorandum of understanding with Foundry $\mathrm{M}$ in India to develop and manufacture the ventilator for the Indian market.

The RespiraWorks team faced unique challenges at the start of the effort. To make an impact in the current pandemic, the development process needed to be significantly shortened from the typical 5-6 years. Thus, the team needed a flexible, efficient approach with multiple designs developed in parallel. Ventilators are inherently a physical system which are often designed using a hardware-focused design - testing iteration loop. With an organization with no physical base of operations and no shared workspace and distributed globally, the challenges of such a hardware-intensive distributed effort were immense. Furthermore, supply chains were significantly disrupted due to the pandemic with medicalgrade parts in short supply. To mitigate these challenges, the team focused on automotive/industrial supply chains, developed custom hardware and sensor solutions using off the shelf parts, and utilized 3D printing for rapid prototyping.

As part of a coordinated hardware and model-based design process, Modelon engaged with the RespiraWorks team to support their efforts. Modelon donated licenses for their new simulation platform Modelon Impact including the Pneumatics Library (Modelon 2021). A team of engineers from Modelon developed pneumatics models of the various design iterations and provided early feedback on design proposals. Simulation was used extensively, especially early in the design process. Using the browser-based Impact platform, Modelon support enabled members of the RespiraWorks team to quickly access the models and execute them to support design iterations.

The following sections in this paper provide an overview of the ventilator modeling effort, including new components developed to support the system design. Pneumatic system models from several design prototypes 
are described along with analysis results from a range of simulations. Lastly an overview of the calibration process is provided along with a comparison of the calibrated model with experimental data from the design prototype developed for the CoVent-19 Ventilator Challenge.

\section{Model Component Overview}

This section provides an overview of the key components of the ventilator model built with the Modelon Pneumatics Library (Modelon 2021). Following a description of a few key components, the system model architecture is shown.

\subsection{Blower}

From the outset, the RespiraWorks team set out to design a full-featured ventilator which did not rely on compressed air, ambu-bag, or mechanical bellows. With medical blowers in short supply and only available at a high cost, the team focused on an automotive/CPAP blower that was readily available through automotive supply chains and able to operate at $1 / 5$ the cost and peak power of a medical blower. Figure 1 shows the X200N 12V DC blower and control board.

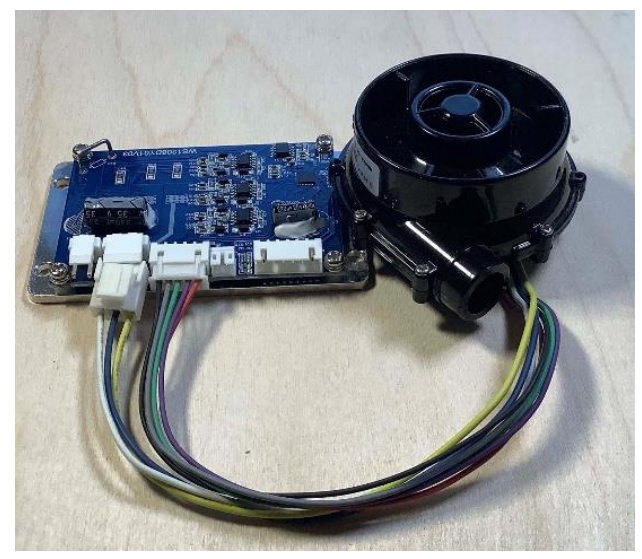

Figure 1. Ventilator blower and control board

A custom blower model was implemented using the fan model from the Modelon library as shown in Figure 2. The fan model includes different options for specifying the flow characteristic based on fan affinity laws. For the X200N model, the table-based characteristic with volume flow rate and power consumption as a function of pressure rise was used. The data provided to characterize the flow is shown in Figure 3 and provides the pressure and volumetric flow but over a range of speeds. Since the table-based model is characterized at a single speed, the table characteristic was tuned to match the data provided resulting in the flow map shown in Figure 4.

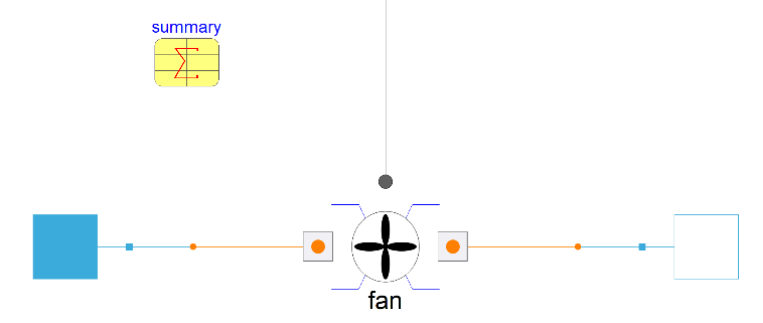

Figure 2. Blower model
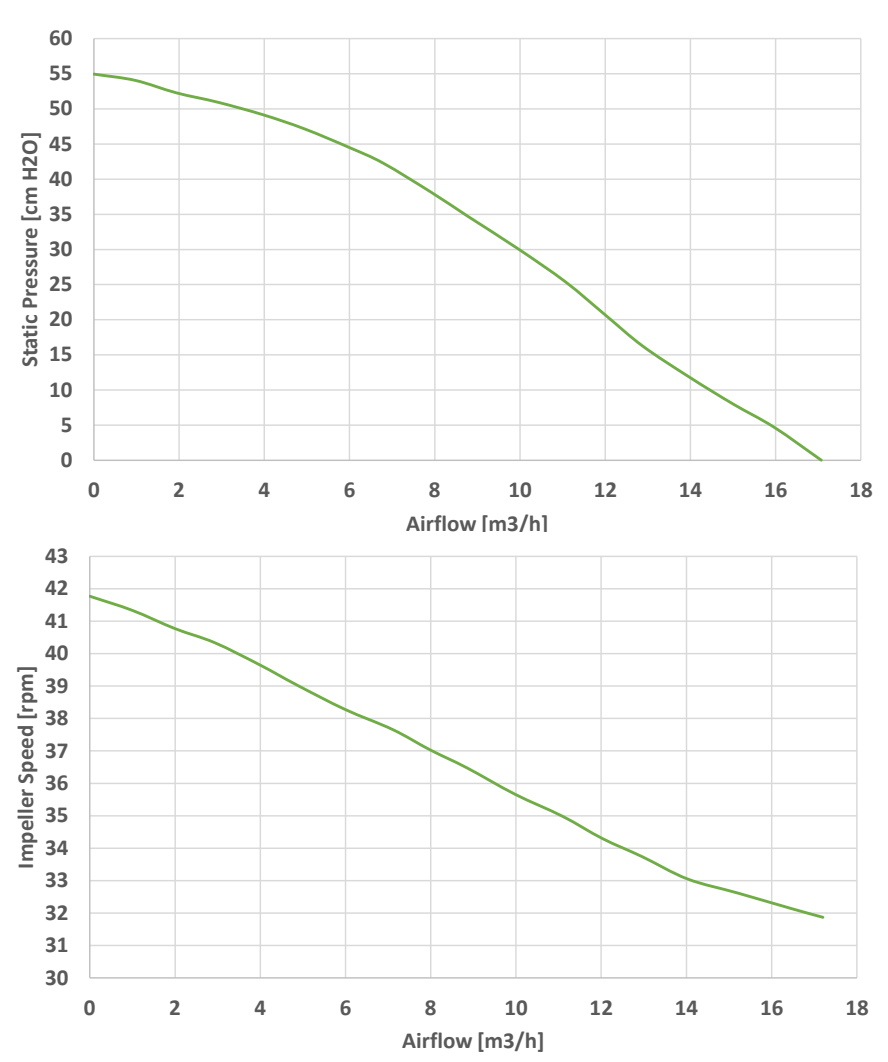

Figure 3. X200N flow characteristics

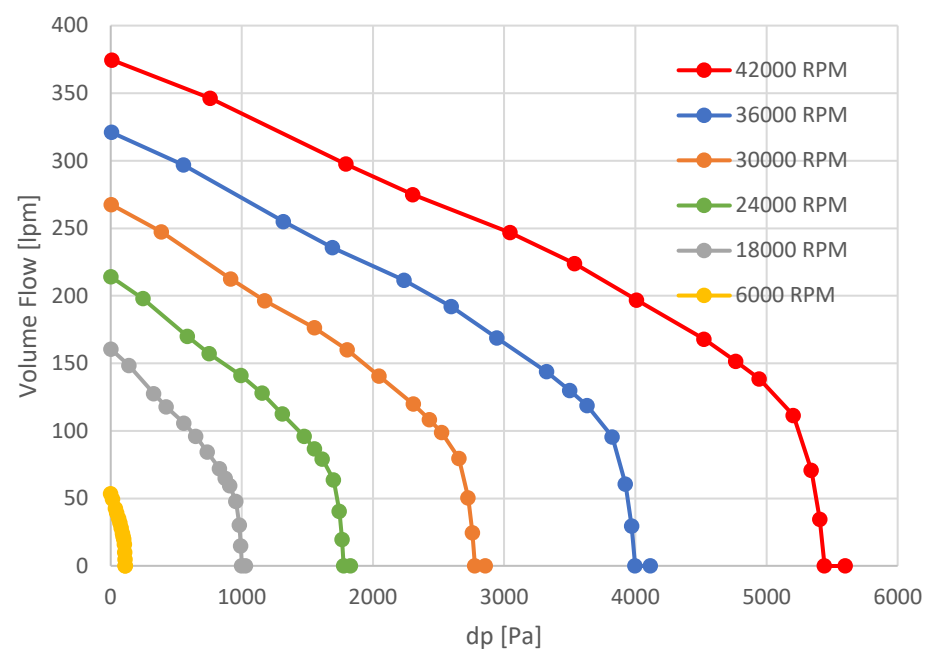

Figure 4. X200N flow map 


\subsection{Venturi Flow Sensor}

Medical flow sensors were completely unavailable at the start of the pandemic. Thus, the RespiraWorks team developed a custom venturi flow sensor using \$20 of commonly available automotive parts to replace the roughly $\$ 500$ cost of unobtainable medical parts. The team arrived at the final design shown in Figure 5 after roughly two dozen iterations. A custom venturi model was implemented to provide the pressure difference based on the characterization performed by the RespiraWorks team. The venturi output pressure difference is provided as a sensor signal to the controller for flow estimation.

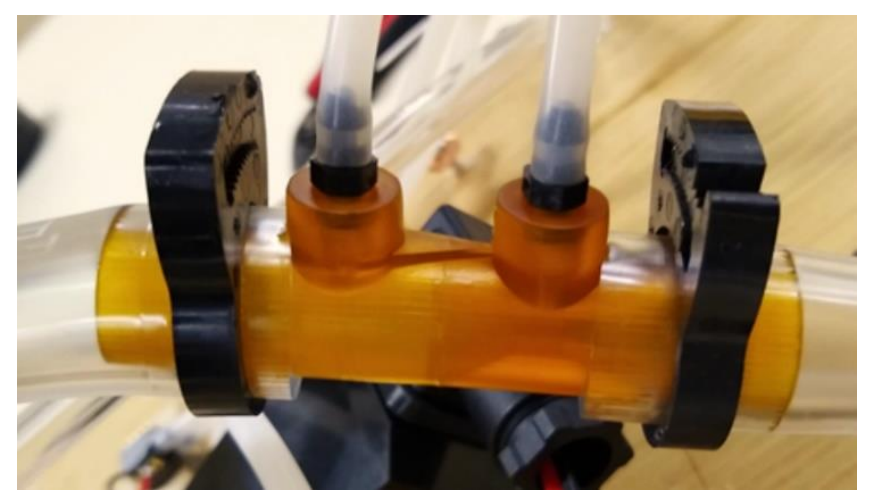

Figure 5. Venturi flow sensor design

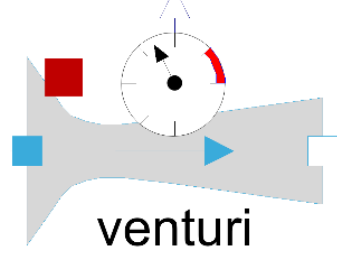

Figure 6. Venturi model

\subsection{Pinch Valve}

Ventilator designs rely on valves to control the air flow during the patient inhale and exhale phases. Many ventilators rely on solenoid valves. Electrical solenoid valves deliver fast response as required at higher breathing rates but at high cost. In addition, proportional solenoids require a high-pressure source to deliver high flow. Thus, using proportional valves on the air side would also require high power blowers or compressors, or the use of hospital-supplied medical compressed air which is not always available. Early simulation work described in Section 3.1 showed that without a fast, high flow valve, the design would require high peak power capability in the blower to reach specified performance targets.

Thus, the team developed a custom pinch valve shown in Figure 7 for flow control. The pinch valve uses an electrically actuated lever to contract the tube, thereby restricting the flow. Though the pinch valve is not capable of completely closing the flow path, it effectively restricts the flow for practical full range flow restriction. The pinch valve was the key innovation that allowed the team to provide a high-flow fast-response flow control without needing to throttle the blower. It is this combination of fast response and low pressure drop at high flow that enabled the use of a commonly-available CPAP blower as the air-side pressure source and also enabled the $5 \mathrm{x}$ reduction in peak blower power by eliminating the need to accelerate and decelerate the blower. To model the pinch valve, a variable table-based flow model was implemented as shown in Figure 8. This model is based on a flow map for the volumetric flow as a function of pressure drop and opening. Based on data provided by the RespiraWorks team, the pinch valve flow map shown in Figure 9 was developed.

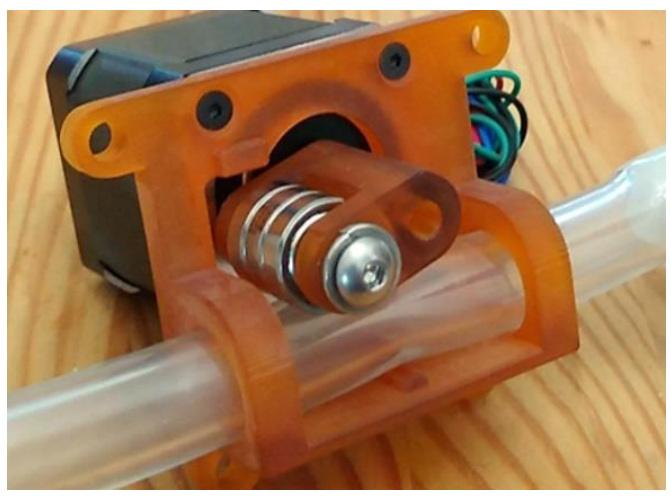

Figure 7. Pinch valve design

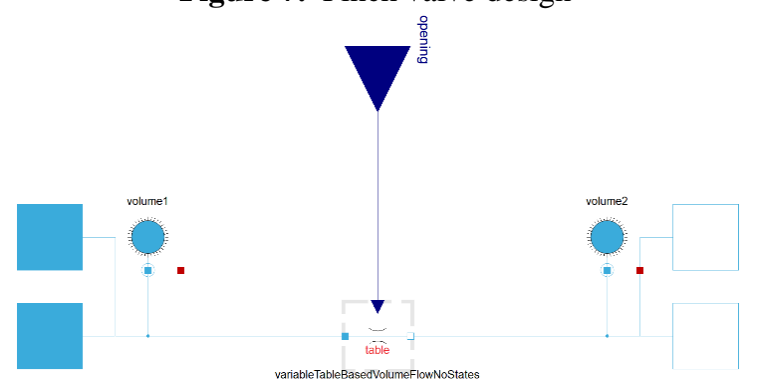

Figure 8. Pinch valve model

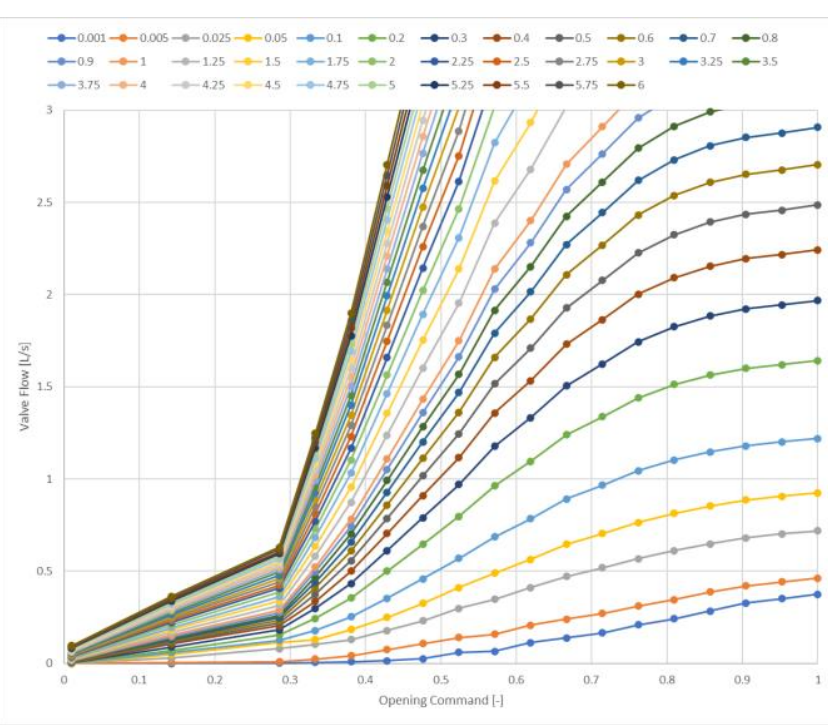

Figure 9. Pinch valve flow map 


\subsection{Proportional Oxygen Valve}

To control oxygen flow to allow a variable ratio of oxygen to air $(\mathrm{FiO} 2)$, the RespiraWorks team designed a system using a proportional oxygen solenoid valve. Figure 10 shows the characterization data for the PVQ30 solenoid valve. This data was used create a map for the variable table-based resistance model. The map is shown in Figure 11. Note that the hysteresis is not considered in the model as a single average between the opening and closing curves was used.

\section{PVQ30 (ø4.0)}

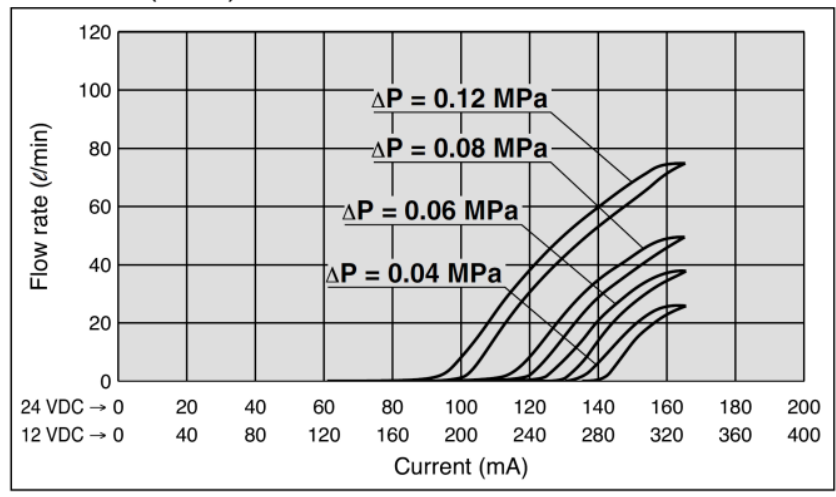

Figure 10. PVQ30 valve characterization data

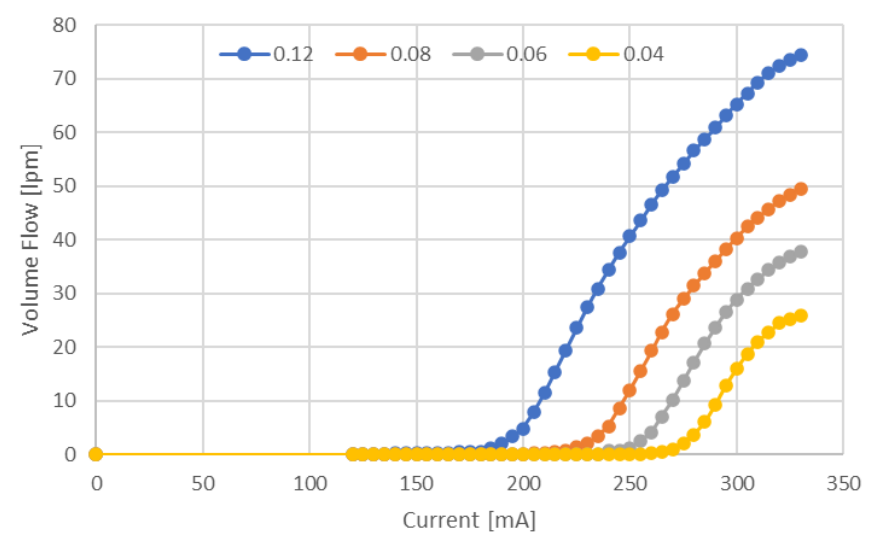

Figure 11. PVQ30 valve data for model

\subsection{Patient Model}

A model of the patient is a critical need for model-based design of the ventilator. The patient model simulates the breathing mechanics of the patient and can be extremely complicated to account for the various resistances and capacitances in the airway, throat, and lung. In addition, the patient model can include the mechanics of the breathing process to account for an active patient (i.e. a patient that can initiate and breathe either partially or completely). Since full-featured ventilators can operate in different modes and with patients requiring varying levels of breathing assistance, the patient model is an important part of the overall model-based development process.
For this work, a simple equation-based model (Arnal 2018) that is typically used to characterize patients was implemented in Modelica. The model includes an overall lung resistance and capacitance and accounts for an active patient with a musculatory pressure term that acts in conjunction with the pressure at the airway opening provided by the ventilator. The model is shown in Figure 12 and relates the lung pressure, volume, and flowrate. The implementation allows for conditional input for the musculatory pressure and also allows the resistance and capacitance to be set via connectors for dynamic response during a simulation or parameters.

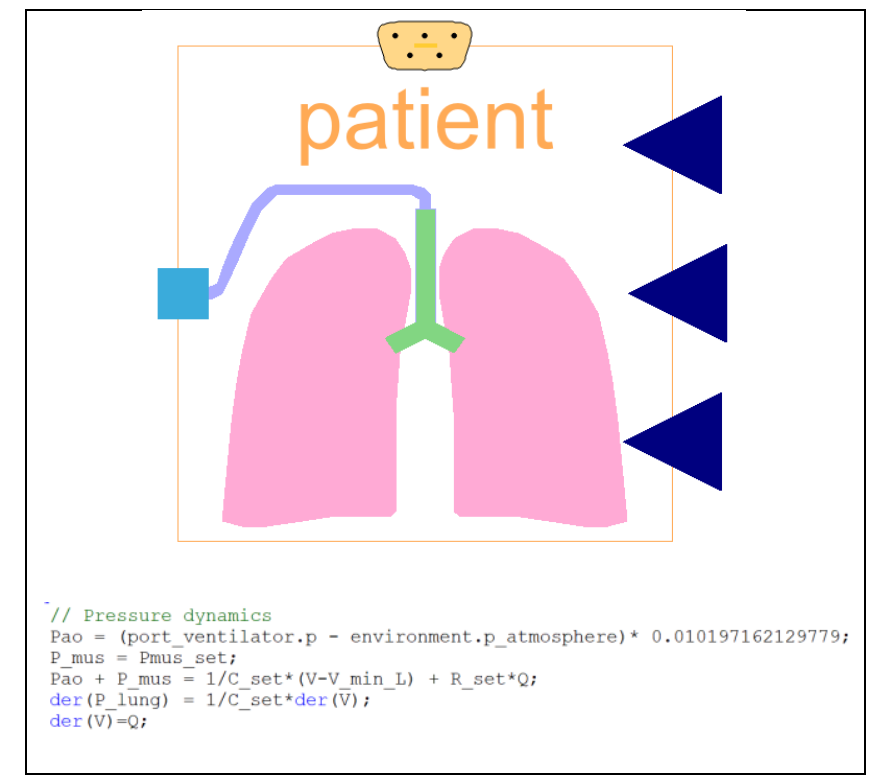

Figure 12. Patient model

\subsection{System Architecture}

Figure 13 shows the system model architecture developed to support model-based development of the ventilator. It consists of three replaceable subsystems:

- Controller

- Ventilator

- Patient

The architecture allows flexible configuration of the various subsystems including implementation of different controllers to support the various ventilator designs. In addition, the architecture supports unit testing of the various components with simplified implementations (i.e. patient test with ventilator as prescribed pressure trace, etc.). Each subsystem is connected via an expandable controlBus to facilitate configuration of the complete system model and also to allow flexibility in the bus variables for each subsystem implementation. Specific implementations of the various subsystems are detailed in the following section. 


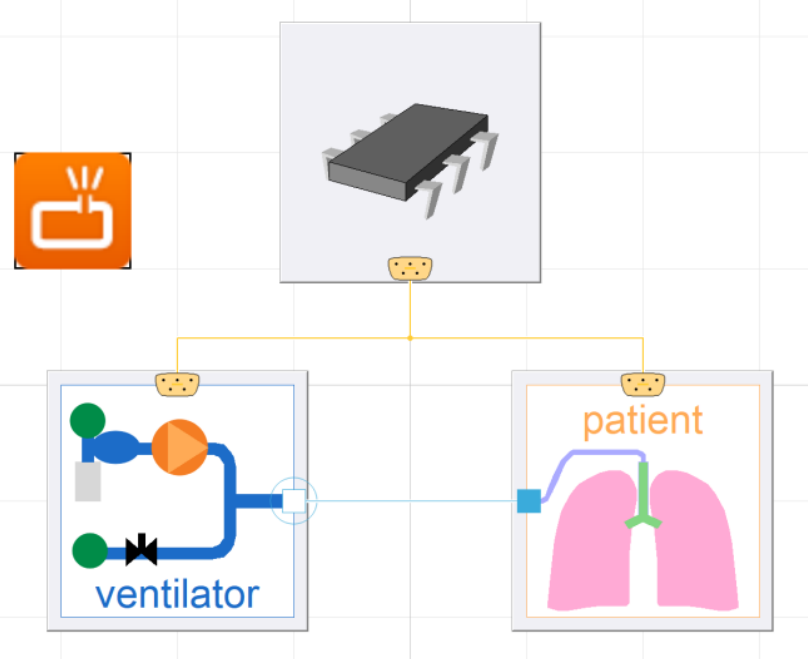

Figure 13. System model architecture

\section{Simulation Results}

To support the model-based design process of the RespiraWorks ventilator, models were implemented of several different ventilator design prototypes. Analysis results from these models were fed back to the RespiraWorks team to support design iterations. This section provides an overview of several of the ventilator designs, associated simulation results, and key findings from the modeling effort to support subsequent design iterations.

The ventilator modeling and simulation was conducted using the new simulation platform Modelon Impact based on the Pneumatics Library (Modelon 2021). Modelon Impact is a next generation system modeling and simulation platform, leveraging the benefits of web and open standard technologies. With openness at its core, Modelon Impact supports standards such as Modelica, FMI, Python and REST (Modelon 2021). The userfriendly browser interface provides modeling experts the tools they need to create, simulate, and experiment. Steady-state or dynamic simulations can be executed from the same model, reducing effort to get an answer (Coïc 2020b) Finally, the Modelon Impact API enables userspecific workflows through Python-based custom functions, and deployment of models to non-experts via targeted web applications or Jupyter Notebooks (Coïc 2020a).

\subsection{Initial Prototype}

Modelon engaged with the RespiraWorks team just as the initial system prototypes were being designed and tested. Figure 14 shows a schematic of the initial prototype design focused on basic hardware prove out and controls requirements. Oxygen is introduced upstream of the blower and mixes with air in a mixing chamber. This design was an early attempt at the system design without any active valve control. This design was meant to assess the feasibility of a design concept where all phases of the breathing process were controlled by the blower and a fixed restriction valve on the exhale limb.

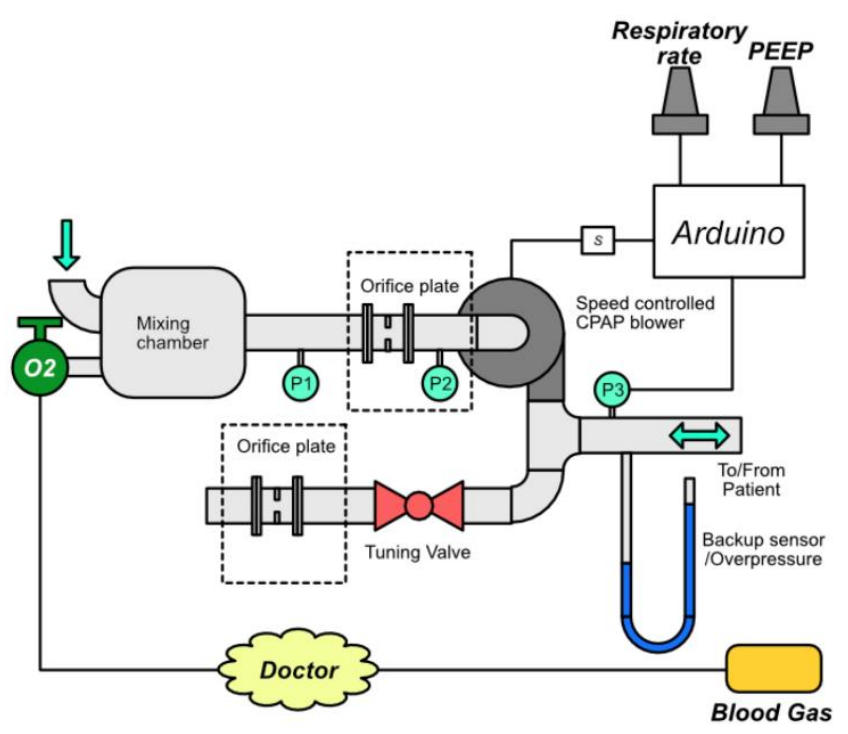

Figure 14. Initial prototype ventilator system

Figure 15 shows the model of the initial prototype in Modelon Impact. The blower speed is controlled to meet a desired pressure $\mathrm{P} 3$ at the patient interface as shown in Figure 16 without any pressure sensor dynamics or noise. Note that the controller architecture includes a component for sensor dynamics, but a null implementation of the sensor dynamic component is used in this controller. In this early phase of development, the desired pressure trace was input directly to the model based on sample traces developed for hardware testing.

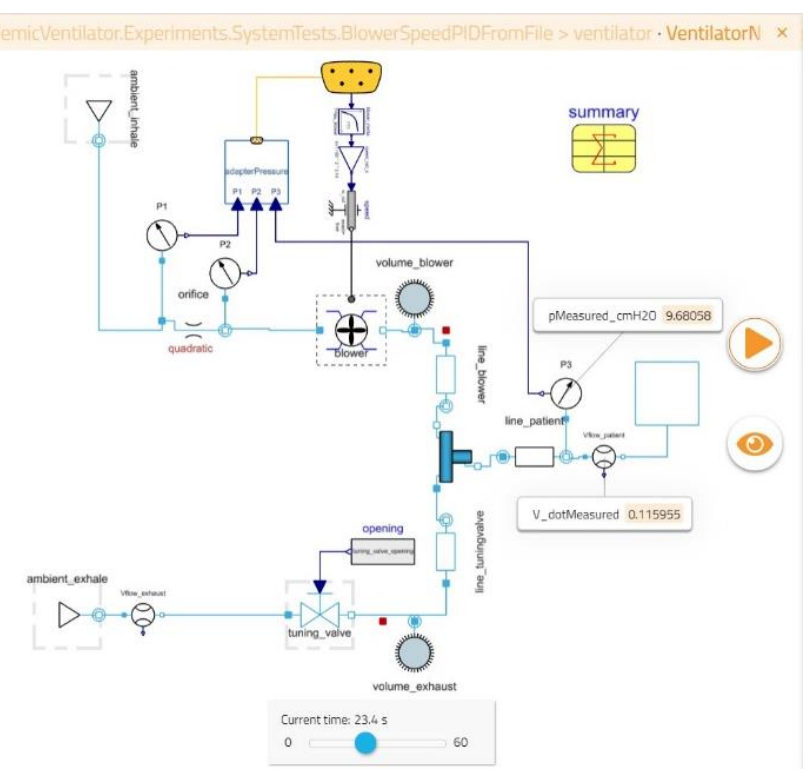

Figure 15. Initial prototype ventilator system model 


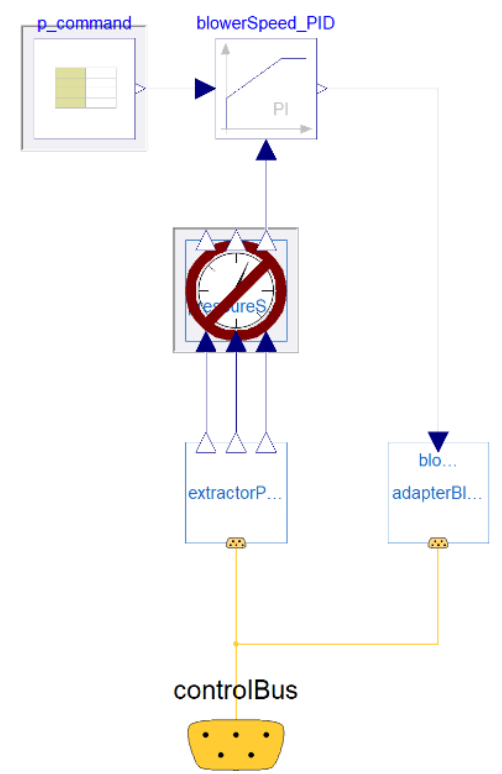

Figure 16. Simple controller for blower speed

Figure 17 shows some initial simulation results integrated with the modeling view while Figure 18 provides a more detailed look at several key results including the pressure response, flowrates, and blower speed. These simulations were run at low breathing rates $(\sim 6$ breaths per minute $)$ and with low pressure targets $(9 \mathrm{~cm} \mathrm{H} 2 \mathrm{O})$. Based on these initial simulations, the following observations were made:
- The blower speed commands indicate the wide operating range required for a single breathing event

- The blower transient response is a concern and the current design would not meet targets for higher breathing rates

- With the current design, the blower transient response would also prohibit higher pressure targets and thus higher flow rates

- Without control valves on the blower and exhaust legs, there is an enormous amount of oxygen waste and excess blower energy consumption as much of the blower flow flows out the exhaust leg as opposed to entering the patient

- It is possible to tune the exhaust resistance via the tuning valve to reduce the oxygen waste during the intake event but at the detriment to patient exhale

- A fixed resistance on the exhaust limb would likely not satisfy requirements over the full range of operating conditions required for the ventilator

These initial simulation results highlighted the importance of the blower transient response to achieve key ventilator targets and also the need for active control valves. The initial modeling work provided crucial feedback to the RespiraWorks team regarding the blower requirements to meet performance targets and led to the subsequent design iterations, including the development of the pinch valve.

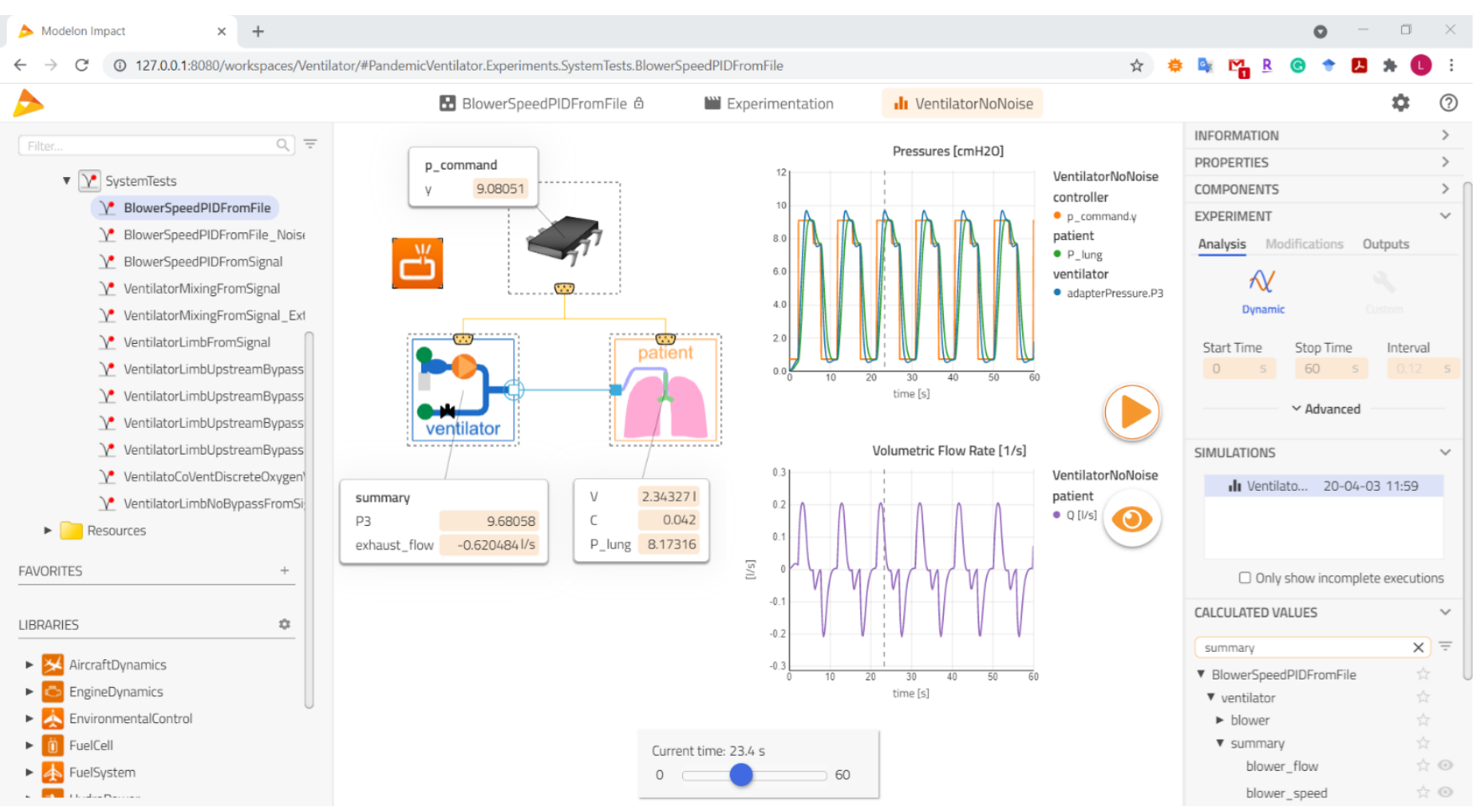

Figure 17. Experiment in Modelon Impact 


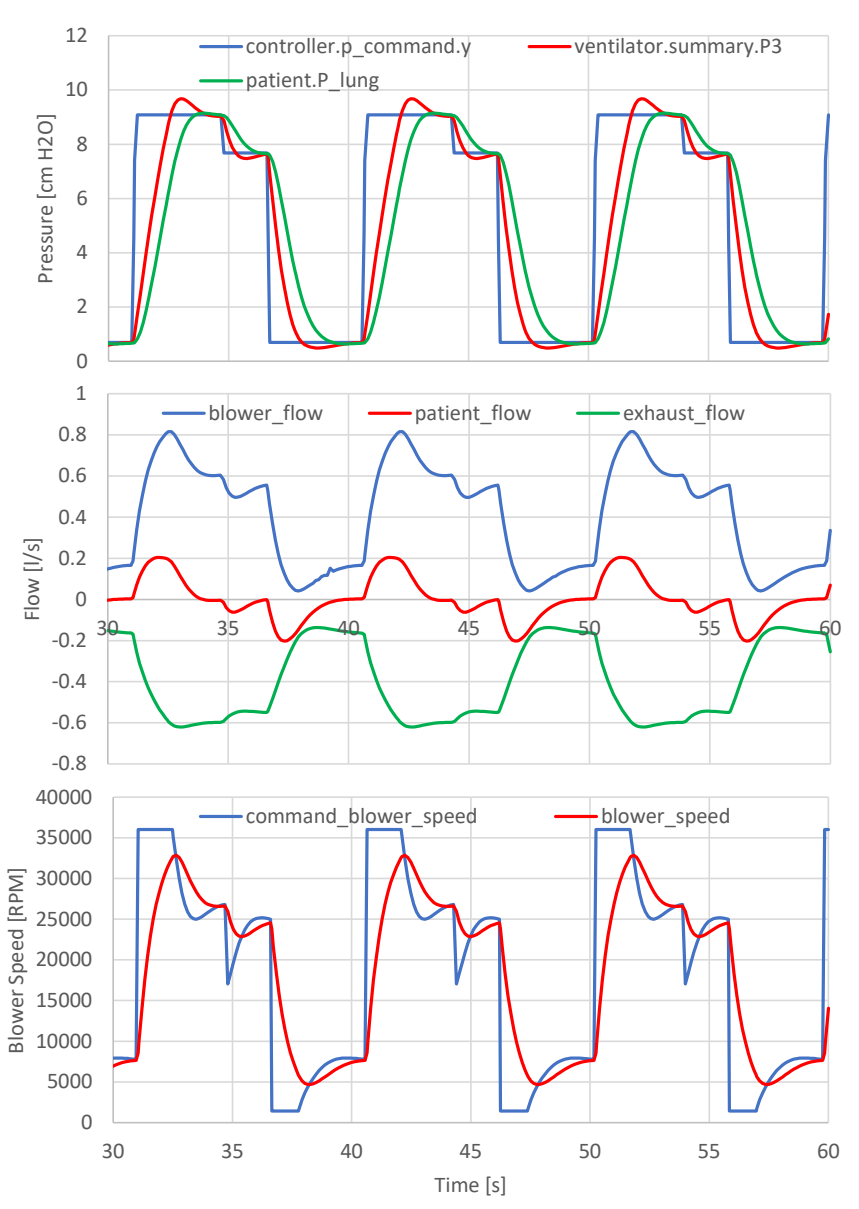

Figure 18. Simulation results for initial prototype

\subsection{Ventilator Mixing Concept}

Based on the results from the initial prototype, the next design iteration was modeled. They key design changes for the ventilator mixing concept shown in Figure 19 include the following:

- Introduction of oxygen downstream of blower as blower is not rated for pure oxygen flow

- Solenoid on exhaust leg

- Check valves upstream of mixing chamber and on intake leg before patient

- Venturi and filter on both intake and exhaust legs

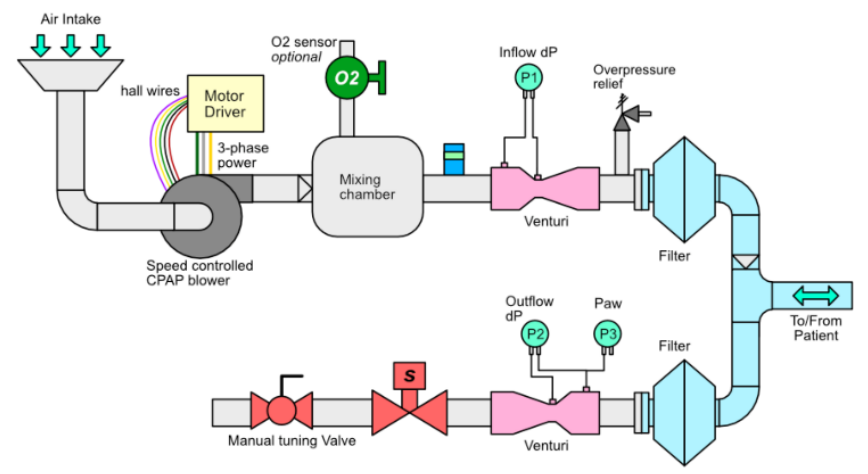

Figure 19. Ventilator mixing concept design
The model for the ventilator mixing concept is shown in Figure 20. This model includes the oxygen source as a prescribed flowrate. The venturi sensors are also included and provide the sensed pressure difference to the control bus. The controller for the model is shown in Figure 21. A custom source block is implemented to provide a pressure command based on parameters for standard ventilator characterization and allows the model to easily run the range of conditions that are required for a fullfeatured ventilator:

- RR: Respiratory rate in breaths per minute

- IE ratio: Inspiration time to exhalation time ratio

- PIP: Peak inspiratory pressure

- PEEP: Positive end expiratory pressure

- Plateau pressure

- Peak to plateau ratio: Peak pressure time to plateau time ratio

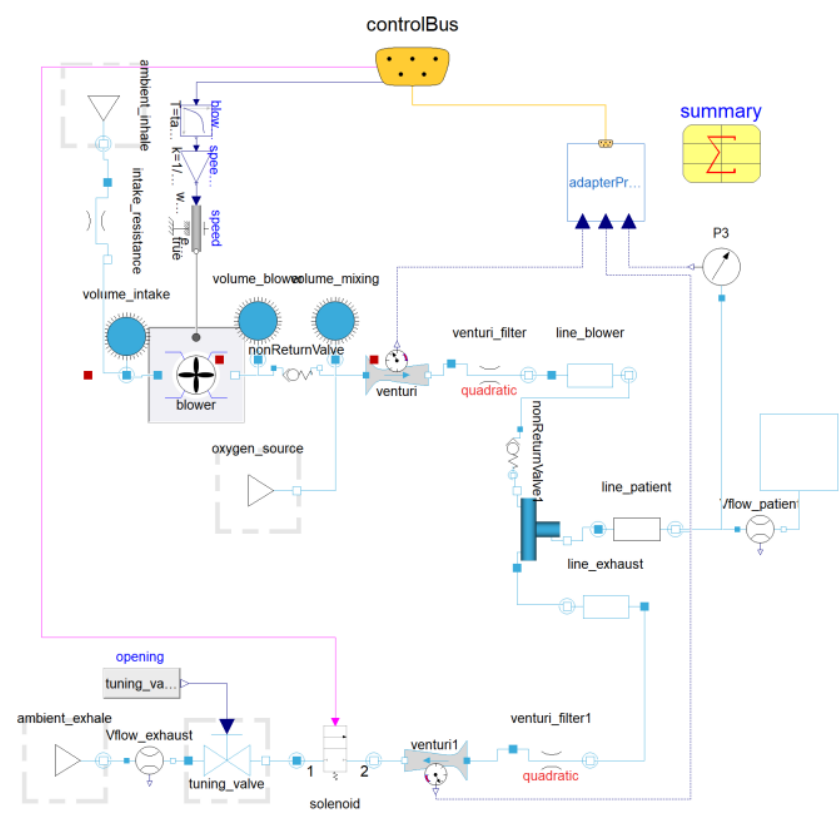

Figure 20. Ventilator mixing concept model

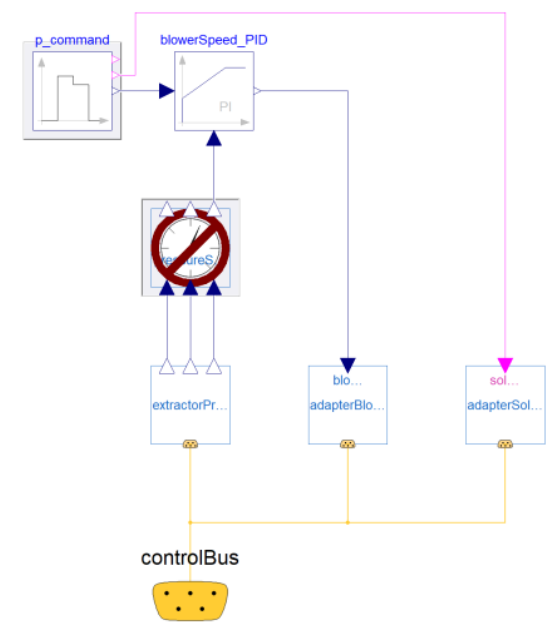

Figure 21. Controller with blower speed and exhaust solenoid based on configurable pressure source 
Figure 22 shows simulation results from the ventilator mixing concept with the pressure command settings at $\mathrm{RR}=20$ breaths $/ \mathrm{min}$, IE ratio $=1 / 2, \mathrm{PIP}=25 \mathrm{~cm} \mathrm{H} 2 \mathrm{O}$, and $\mathrm{PEEP}=6 \mathrm{~cm} \mathrm{H} 2 \mathrm{O}$ for a patient with $\mathrm{R}=13 \mathrm{~cm} \mathrm{H} 2 \mathrm{O} \mathrm{s} / \mathrm{L}$, $\mathrm{C}=0.042 \mathrm{~L} / \mathrm{cm} \mathrm{H} 2 \mathrm{O}$. These results indicate the following:

- Solenoid eliminates exhaust flow during intake event and reduces oxygen waste

- Even with relatively slow blower transient response, ventilator is just able to meet PIP target at higher breathing rates since exhaust is closed during intake event

- Large flow when solenoid opens (roughly equal to blower flow as blower speed has not reduced plus flow out from dead exhaust volume) helps reduce pressures and facilitate exhale

- Excess exhaust flow from intake (blower + oxygen) until blower speed drops with blip at start of exhaust event due to reduced back pressure

- Blower speed increases around middle of exhaust event to maintain PEEP level

These simulations indicate that the design is significantly improved from the initial prototype and highlight the importance of coordinated valve control along with pneumatic system design.

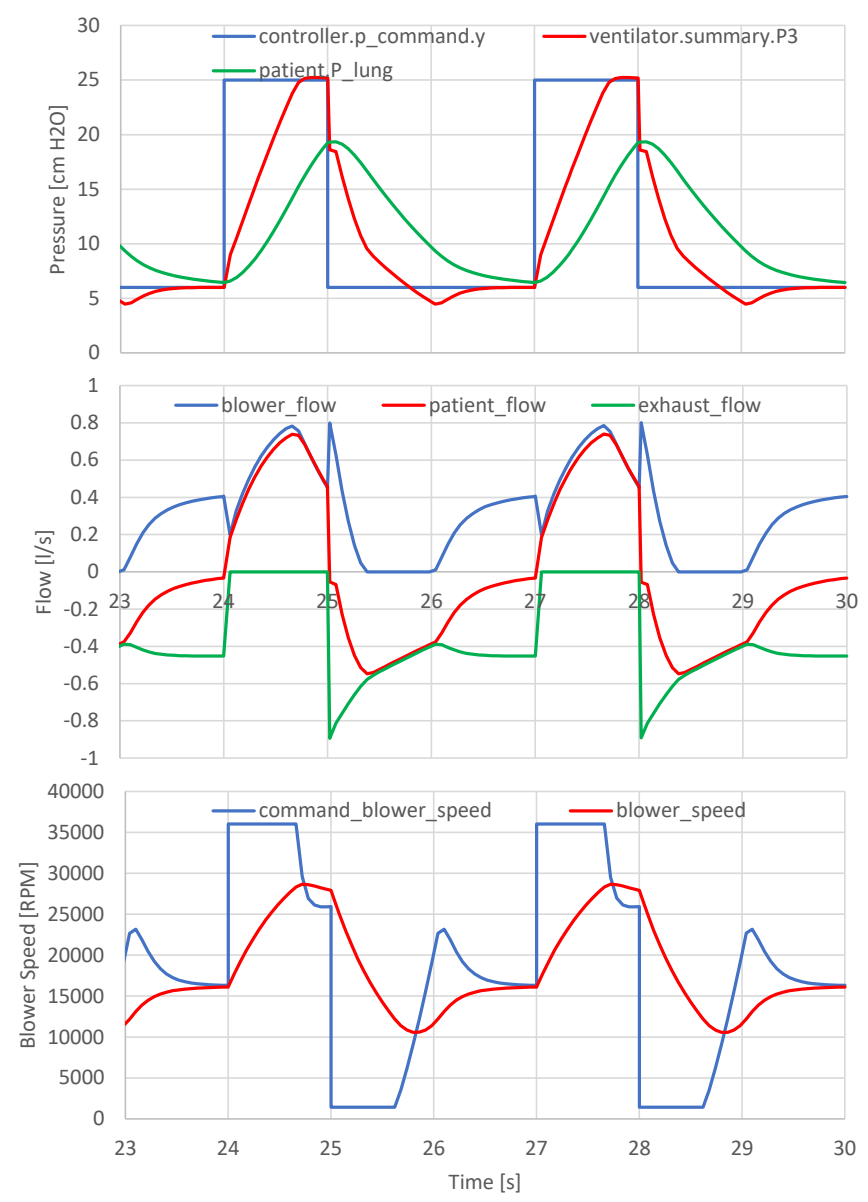

Figure 22. Simulation results for ventilator mixing concept

\subsection{CoVent Concept}

The next design modeled was the CoVent concept design shown in Figure 23. This design represents design intent for the CoVent-19 Ventilator Challenge. The design updates include the following:

- Addition of closed loop oxygen control via the oxygen proportional solenoid valve and pressurized oxygen source

- Intake blower and exhaust pinch valves

This concept provides full control of the air, oxygen, and exhaust flows via the various valves and allows for closed loop control of oxygen to meet a range of oxygen to air ratios.

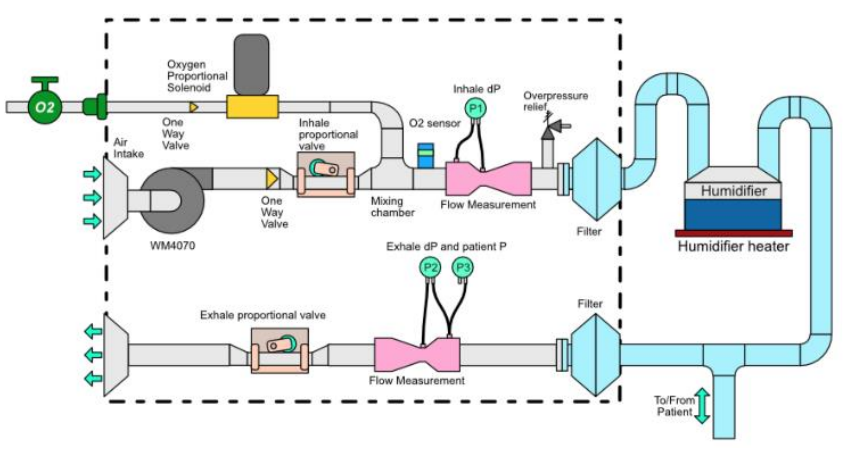

Figure 23. CoVent concept design

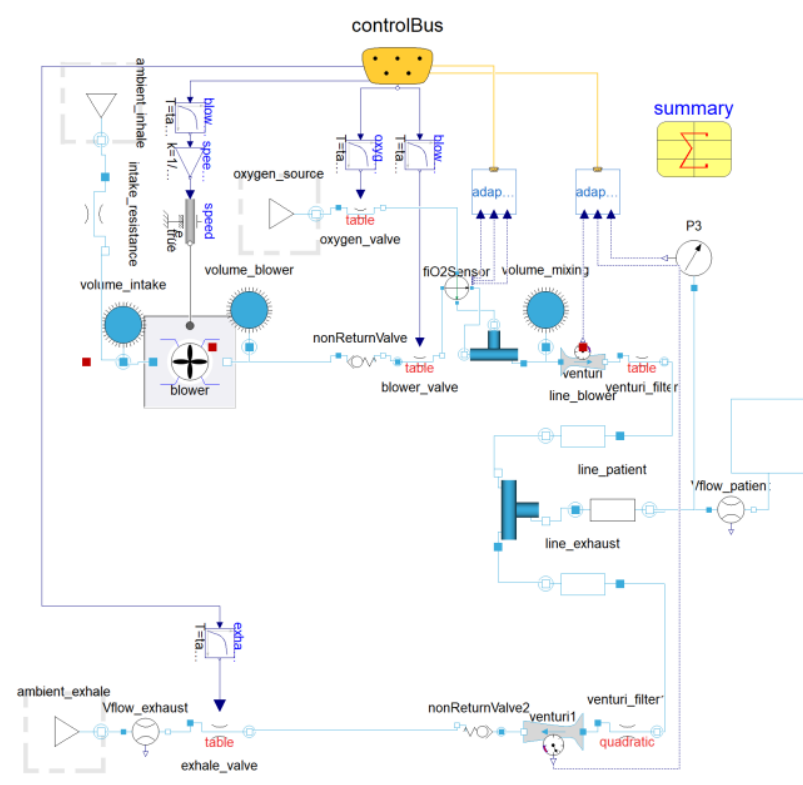

Figure 24. CoVent design model

Along with the modeling effort, the hardware was being developed for testing to support the CoVent-19 Challenge submission. Figure 25 shows the hardware realization, including the QuickLung to simulate the patient. Data was taken on the hardware over the range of operating conditions outlined in green in Table 1. These tests were run with air only and also with oxygen only as the closed 
loop $\mathrm{FiO} 2$ controller was not complete when the tests were run. This data was made available for model calibration. The data includes the pressure control setpoint, recorded pressure, inhale and exhale venturi pressure difference, inhale and exhale pinch valve command, and oxygen valve command.

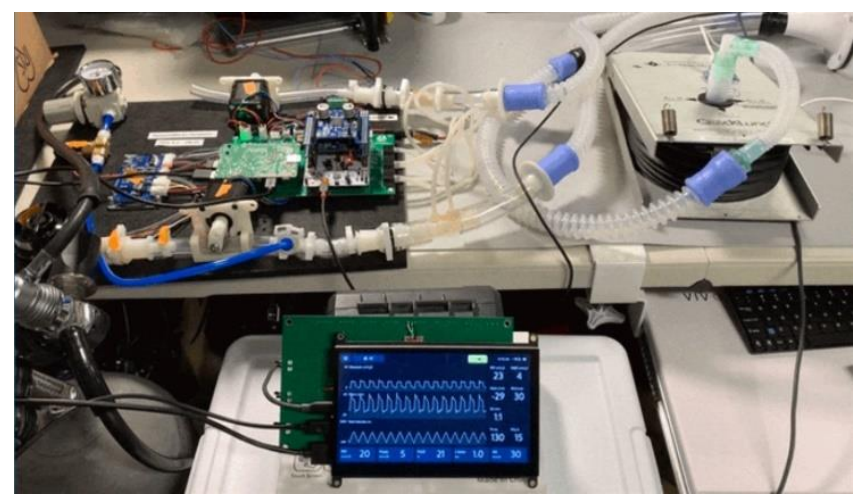

Figure 25. CoVent hardware realization

Table 1. Test settings for CoVent runs Table 2: Section 201.12.1.101 from ISO 80601-2-12

Table 201.104 - Volume-control inflation-type testing

\begin{tabular}{|c|c|c|c|c|c|c|c|}
\hline \multirow[b]{2}{*}{$\begin{array}{c}\text { Test } \\
\text { number }\end{array}$} & \multicolumn{2}{|c|}{ Test lung parameters } & \multicolumn{5}{|c|}{ Ventilator settings } \\
\hline & $\begin{array}{c}\text { Compliance } \\
\mathrm{ml} / \mathrm{hPa} \\
\pm 10 \%\end{array}$ & $\begin{array}{c}\text { Linear } \\
\text { resistance[1[1][18][19] } \\
\mathrm{hPa} / 1 / \mathrm{s} \\
\pm 10 \%\end{array}$ & $\begin{array}{c}\text { Tidal } \\
\text { volume } \\
\mathrm{ml}\end{array}$ & $\begin{array}{c}\text { Set ratea } \\
\text { breaths/min }\end{array}$ & $\begin{array}{c}\text { Inspiratory } \\
\text { time } \\
\mathrm{s}\end{array}$ & $\begin{array}{l}\mathbf{O}_{2} \\
\%\end{array}$ & $\begin{array}{c}B A P \\
\mathrm{hPa} \\
\left(\mathrm{cmH}_{2} \mathrm{O}\right)\end{array}$ \\
\hline 1 & 50 & 5 & 500 & 20 & 1 & 30 & 5 \\
\hline 2 & 50 & 20 & 500 & 12 & 1 & 90 & 10 \\
\hline 3 & 20 & 5 & 500 & 20 & 1 & 90 & 5 \\
\hline 4 & 20 & 20 & 500 & 20 & 1 & 30 & 10 \\
\hline 5 & 20 & 20 & 300 & 20 & 1 & 30 & 5 \\
\hline 6 & 20 & 50 & 300 & 12 & 1 & 90 & 10 \\
\hline 7 & 10 & 50 & 300 & 20 & 1 & 30 & 10 \\
\hline 8 & 10 & 10 & 200 & 20 & 1 & 90 & 5 \\
\hline 9 & 3 & 10 & 50 & 30 & 0,6 & 30 & 5 \\
\hline 10 & 3 & 20 & 50 & 30 & 0,6 & 30 & 10 \\
\hline 11 & 3 & 50 & 50 & 20 & 0,6 & 60 & 5 \\
\hline 12 & 3 & 20 & 30 & 30 & 0,6 & 30 & 5 \\
\hline 13 & 3 & 50 & 30 & 20 & 0,6 & 90 & 10 \\
\hline 14 & 1 & 20 & 30 & 30 & 0,6 & 90 & 5 \\
\hline 15 & 1 & 100 & 30 & 30 & 0,6 & 30 & 10 \\
\hline 16 & 1 & 200 & 20 & 50 & 0,4 & 30 & 5 \\
\hline 17 & 1 & 200 & 15 & 50 & 0,4 & 60 & 10 \\
\hline 18 & 1 & 50 & 10 & 60 & 0,4 & 60 & 5 \\
\hline 19 & 0,5 & 50 & 5 & 60 & 0,4 & 60 & 10 \\
\hline 20 & 0,5 & 200 & 5 & 30 & 0,4 & 30 & 5 \\
\hline 21 & 0,5 & 200 & 5 & 60 & 0,4 & 30 & 10 \\
\hline
\end{tabular}

To facilitate running the model based on experimental data for calibration and validation, the controller shown in Figure 26 was developed. This controller reads the time traces from the experimental data and provides them for input to the model. In this design, the blower is always run at max speed and the pinch valves are used to control the intake and exhaust events along with the oxygen valve. The valve commands are output from the experimental data and used to drive the simulations. After running the initial simulations driven by the experimental data, it was readily apparent that the valve commands did not match with the characterization data provided for the valves.
After consulting with the RespiraWorks controller development team, the source of the difference was identified as a feature in the controller that performs a system calibration procedure to identify the min and max operating point for the pinch valve and oxygen valve. In addition, the controller includes a linearization table that maps the valve command to the characterized valve position shown in Figure 9 for a linear response in flow. These tables were also implemented in the controller shown in Figure 26 but were allowed to change in the model for calibration purposes.

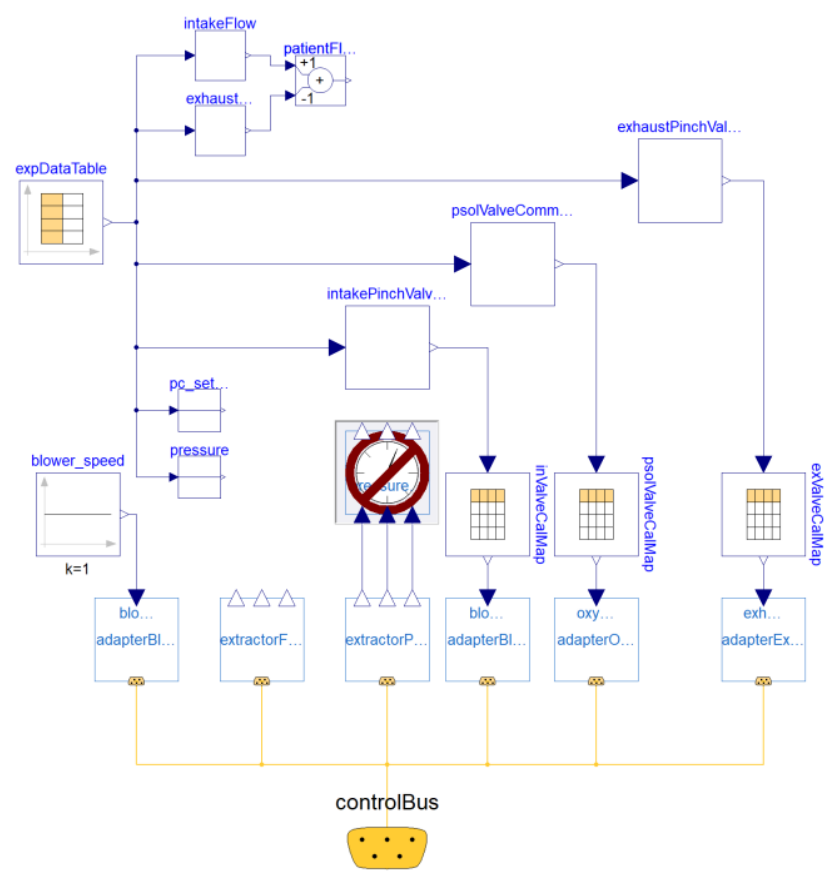

Figure 26. Controller for calibration/validation

The overall calibration procedure for the model is as follows:

- Run model with input flow rate and exhaust valve active to tune overall system resistance and exhaust pinch valve mapping

- Run model with all valves active and adjust blower pinch valve and oxygen valve mapping

- Adjust time constants based on dynamic results

- Re-run all tests for validation

The goal of this calibration procedure is to produce a model of the ventilator system that accurately reproduces the response from the CoVent hardware and can be used to support controller design and calibration. Note the following regarding the calibration approach:

- The blower map was not adjusted

- System response from open loop controller inputs provided as input to the model are difficult to match 
- Differences between the simulation and data will exist, especially at steady state

- Overshoot in pressures will persist since controller inputs are provided open loop

- Calibration goal is to get good match to pressures, flowrates, and overall transient response for a range of operating conditions since closed loop controller would improve pressure tracking

Figure 27 - Figure 31 (included at the end of the paper for formatting reasons) compare simulation results with experimental data recorded from the CoVent setup. Each figure compares flow rates and pressures for a specific test condition described in Table 1. Experimental data is indicated in the legend with the "(exp)" label. In the flow plot comparison, the intake and exhaust experimental flow is compared with the blower flow, oxygen flow, and exhaust flow from the model. Since the experimental data was obtained with air only or oxygen only, one of the modeled flow signals will be zero for a given test. In the pressure plot comparison, the pressure setpoint is shown along with the experimental patient pressure from the QuickLung and the model equivalent pressure labeled P3.

Figure 27 shows simulation results from Test 1 with input air flowrate after tuning the exhaust pinch valve mapping and overall system resistance (i.e. components for which no flow characterization was provided). There is excellent agreement in the pressure response when the flowrate is provided as an input in the calibrated model. The results provide validation that the venturi calculation, exhaust valve mapping, and overall system resistance are appropriate.

Figure 28 - Figure 31 show results from different tests in Table 1 with oxygen and air. In these tests, the full model predictions for flowrates and pressures are exercised based on the modeled controller shown in Figure 26. These tests are run with input traces from the experimental data for the intake pinch valve command, oxygen valve command, and exhaust pinch valve command in the modeled controller with resulting model flows.

In general, the results show good agreement with the experimental data. The following observations can be made:

- Overall response for oxygen only runs looks reasonable

- Hysteresis effects (Figure 10) from the oxygen valve are seen in data but not in model as model overpredicts flow decrease during closing command

- Overall response for air only runs looks reasonable

- Intake flow overpredicted a bit which could be attributed to the blower map

- Model results are consistent with higher flowrates resulting in pressure overshoot

\section{Summary}

This paper describes the model-based development of the open source RespiraWorks ventilator. Using the Modelon Pneumatics Library in the Modelon Impact platform, various design iterations of the ventilator were modeled. The ventilator designs were tested in a configurable system architecture in conjunction with controller implementations and a model of the patient. The RespiraWorks ventilator design for the CoVent-19 Ventilator Challenge was modeled and calibrated using experimental data collected to support the challenge submission. The calibrated model showed good agreement with experimental data. Future work with the model will focus on the application of the model for controller design and tuning.

The RespiraWorks team finished $3^{\text {rd }}$ in the CoVent-19 Ventilator Challenge and is continuing their effort to design and build their ventilator. For the latest updates on the design process, visit the repository at https://github.com/RespiraWorks/Ventilator

\section{Acknowledgements}

The authors would like to thank Anand Pitchaikani and Midhun Joy from Modelon for their contribution to the model development and Impact deployment at RespiraWorks. The authors also extend their sincere thanks to the entire RespiraWorks team, particularly David Gershon, for his engagement with the modeling effort.

\section{References}

Arnal, Jean-Michel, Aude Garnero, Mathieu Saoli, and Robert Chatburn (2018). "Parameters for Simulation of Adult Subjects During Mechanical Ventilation”. In: Respiratory Care. February 2018. Vol. 63. No. 2. pp. 158-168. DOI: 10.4187/respcare.05775.

Coïc C., J. Andreasson, A. Pitchaikani, J. Åkesson, and H. Sattenapalli (2020), "Collaborative Development and Simulation of an Aircraft Hydraulic Actuator Model", Asian Modelica Conference, Tokyo, Japan.

Coïc C., M. Hübel, and M. Thorade (2020), "Enhanced SteadyState in Modelon Jet Propulsion Library, an Enabler for Industrial Design Workflows". American Modelica Conference 2020, Boulder, Colorado, USA,

Modelon (2021). Impact. URL: https://www.modelon.com/modelon-impact/.

Modelon (2021). Pneumatics Library. URL: https://www.modelon.com/library/pneumatics-library/. RespiraWorks (2021). URL: https://www.respiraworks.com. 


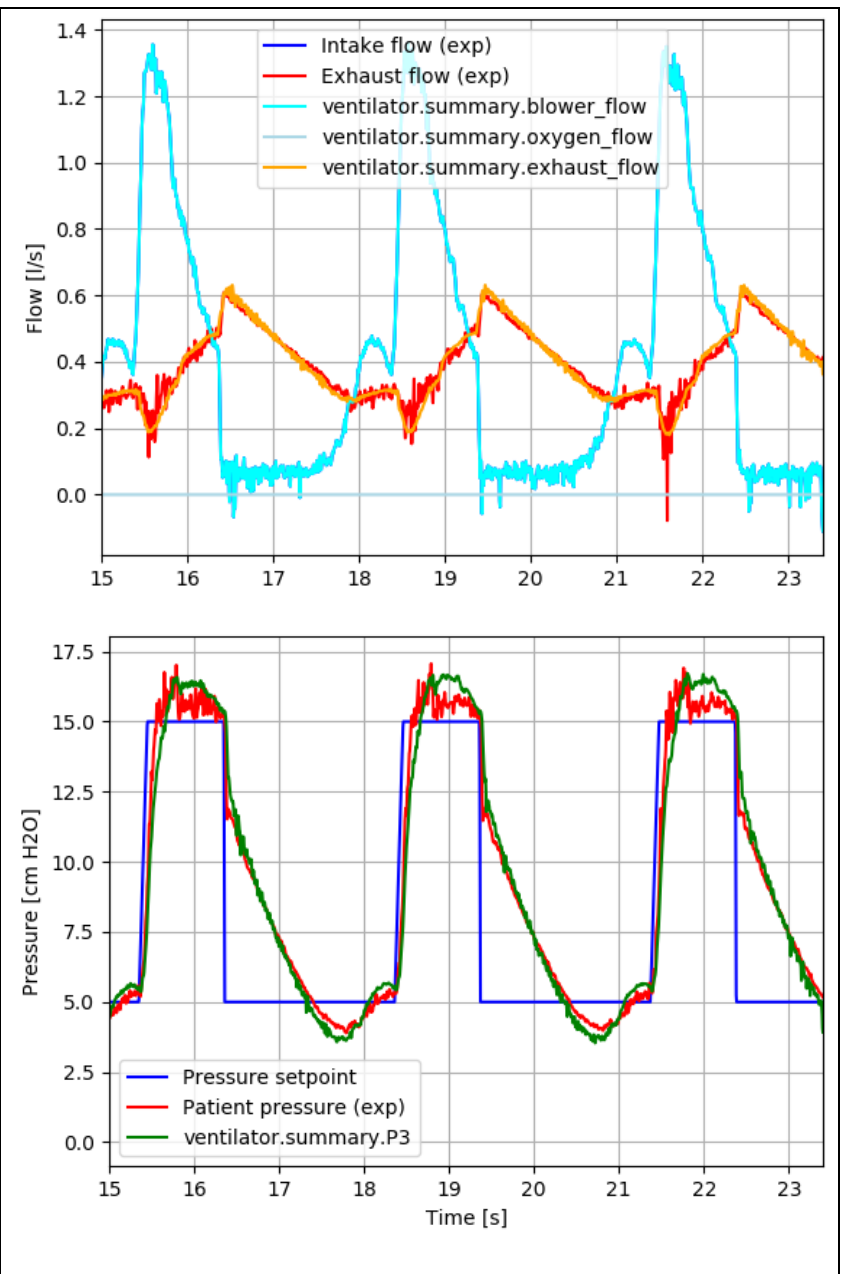

Figure 27. Simulation results from Test 1, air only, input flowrate
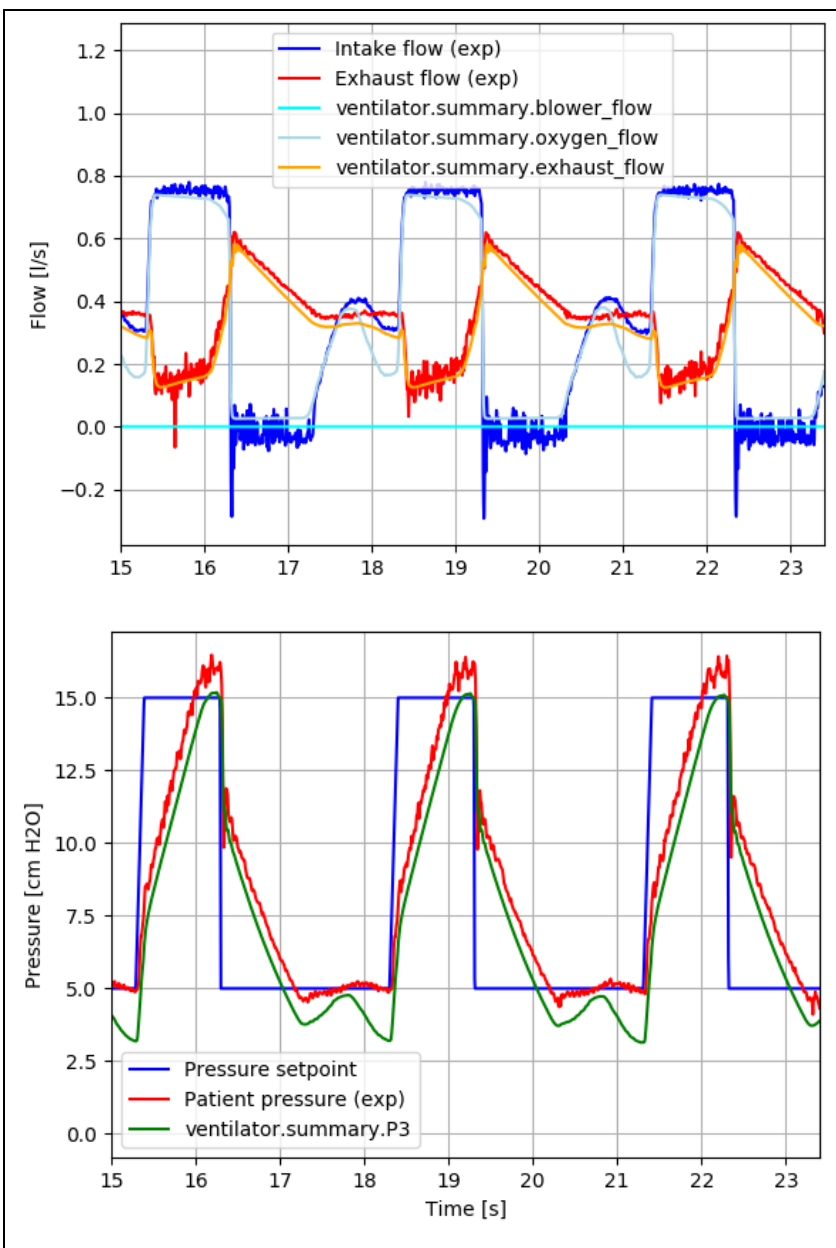

Figure 28. Simulation results from Test 1, oxygen only 


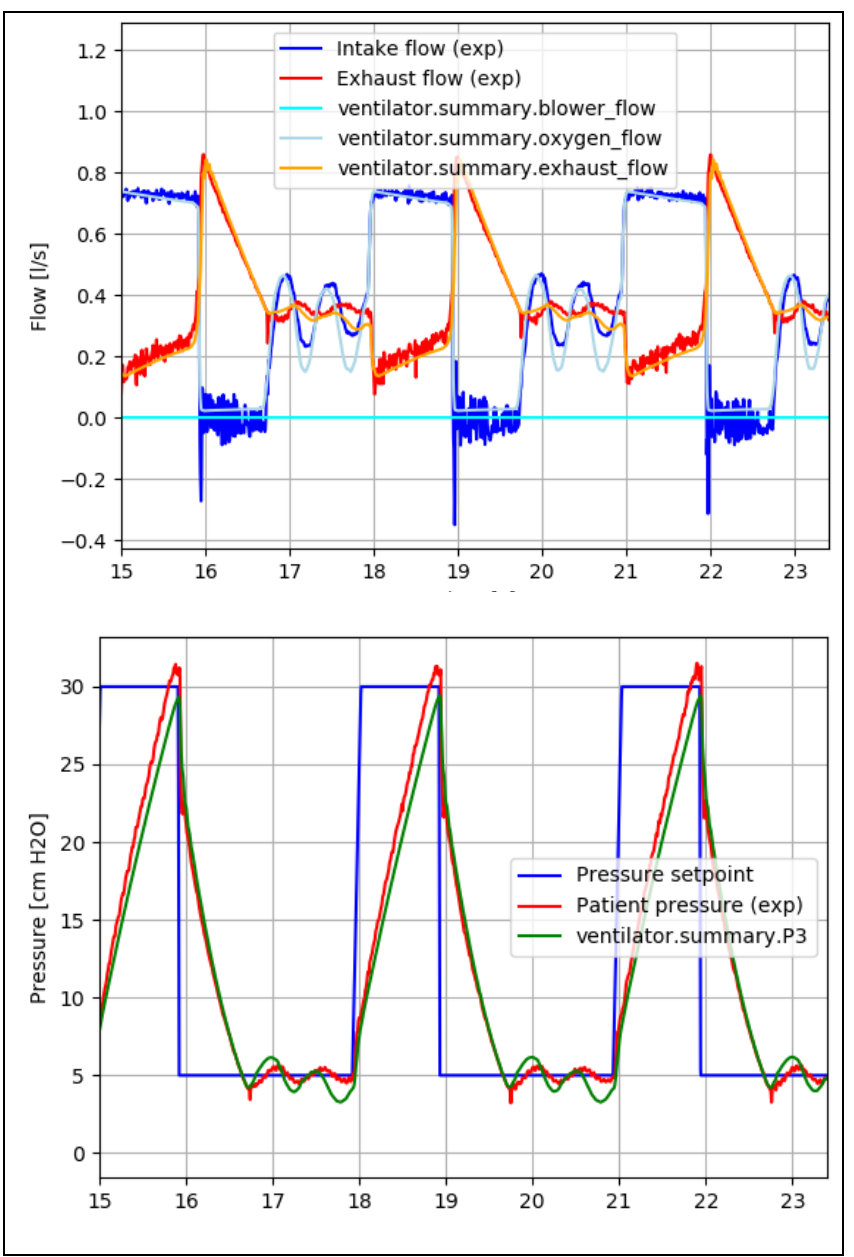

Figure 29. Simulation results from Test 3, oxygen only
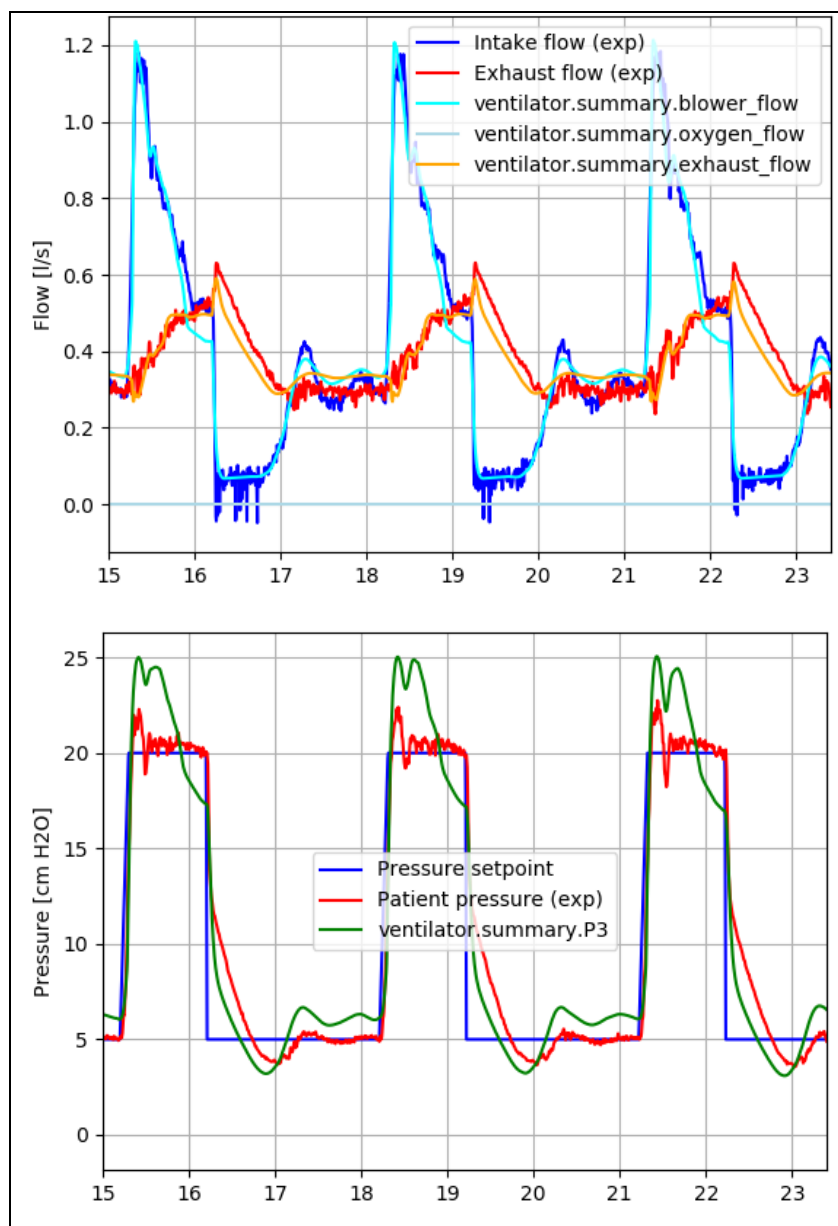

Figure 30. Simulation results from Test 5, air only 


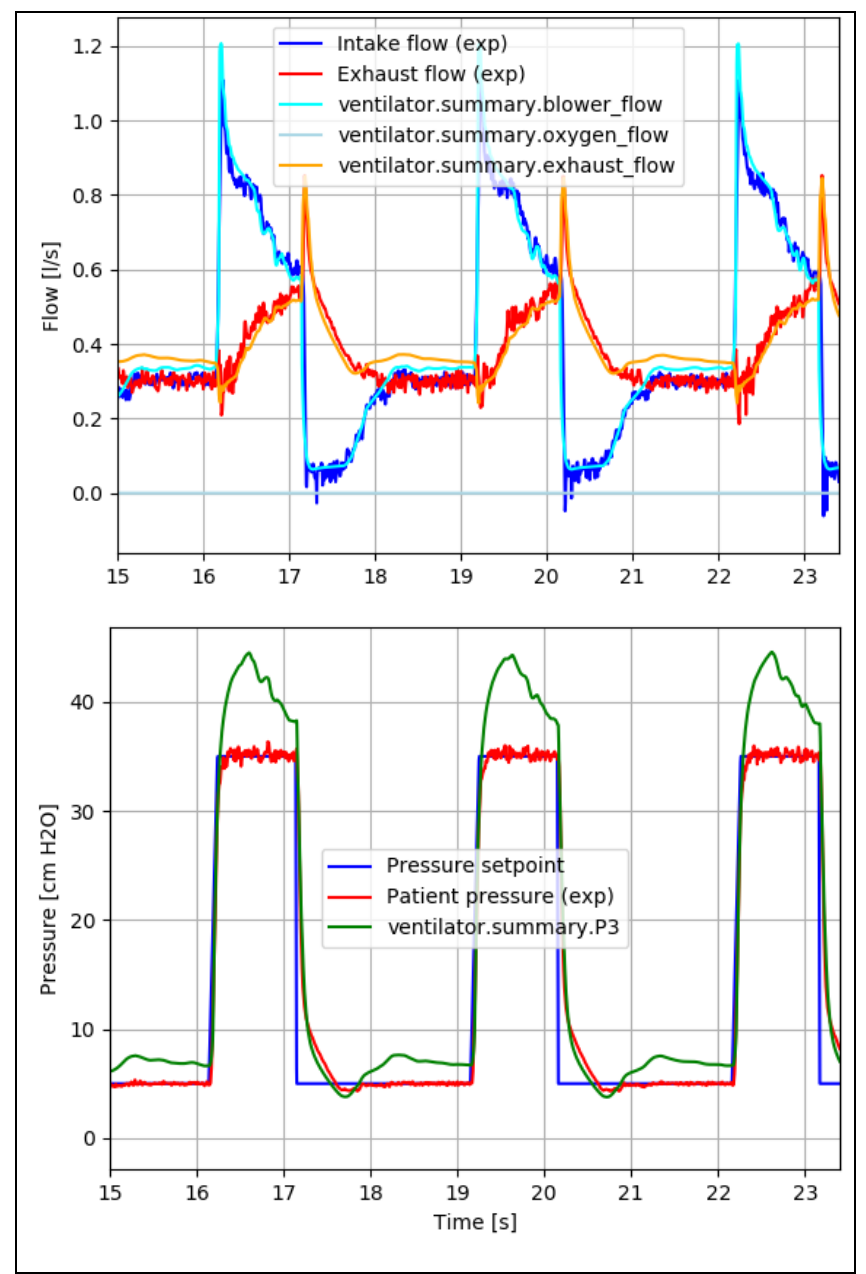

Figure 31. Simulation results from Test 7, air only 